\title{
Un inusual caso de granuloma piogeno oral multiple, ¿asociado a tratamiento con levotiroxina?
}

An unusual case of multiple oral pyogenic granuloma, ;associated with treatment with levothyroxin?

Alejandro Arturo Gutiérrez Patiño-Paul 1,a

\section{RESUMEN}

El Granuloma piógeno (GP) es una lesión tumoral, no neoplásica, vascular, generalmente solitaria, que se presenta en la cavidad oral o piel, la etiología aún no está muy clara, se considera que es una lesión reactiva a varios estímulos de bajo grado, entre los cuales podemos contar traumas repetidos, agresiones, factores hormonales y ciertos fármacos. El GP múltiple, es un fenómeno raro, generalmente asociado al uso de drogas, como la carbamacepina, ciclosporina, retinoides, levotiroxina, etc. Se presenta el caso de una paciente de 50 años, referida por presentar aumento de volumen en reborde alveolar del primer, tercer y cuarto cuadrantes; con antecedentes de psoriasis eritrodermica e hipotiroidismo, en tratamiento con levotiroxina aproximadamente seis meses antes que se presenten las lesiones; se realiza exeresis quirúrgica y curetaje subperiostico, se enviaron las muestras a anatomía patológica, confirmándose el diagnóstico definitivo de GP; por los antecedentes, y la aparición casi simultánea de las lesiones en múltiples cuadrantes, se sospecha que estas, están asociadas al consumo terapéutico de levotiroxina para el hipotiroidismo.

PALABRAS CLAVE: Granuloma piógeno, hipotiroidismo, levotiroxina. 


\section{SUMMARY}

Pyogenic Granuloma (GP) is a tumorous, non-neoplastic, vascular, generally solitary lesion that presents itself in the oral cavity or skin, the etiology is still not very clear, it is considered to be a reactive lesion to several low grade stimuli, among which we can count repeated trauma, aggressions, hormonal factors and certain drugs. The multiple GP, is a rare phenomenon, generally associated with the use of drugs, such as carbamacepine, cyclosporine, retinoids, levothyroxine, etc. It presents the case of a patient of 50 years, referred for presenting increased volume in alveolar ridge of the first, third and fourth quadrants, with a history of erythrodermic psoriasis and hypothyroidism, in treatment with levothyroxine approximately six months before the lesions occur; Surgical exeresis and subperiosteal curettage is performed, samples were sent to pathological anatomy, confirming the definitive diagnosis of GP; because of the history, and the almost simultaneous appearance of the lesions in multiple quadrants, it is suspected that these are associated with the therapeutic consumption of levothyroxine for hypothyroidism.

KEYWORDS: Pyogenic granuloma, hypothyroidism, levothyroxine.

\section{INTRODUCCION}

El Granuloma piógeno (GP) es una lesión tumoral, no neoplásica, vascular, reactiva, generalmente solitaria, que se presenta en la cavidad oral o piel. Ha sido conocido con una diversidad de nombres, como Botryomycosis hominis, Botryomycoma, Granuloma telangiectásico, Granuloma benigno pedunculado, Pseudobotryomycosis, Fibroangioma, Enfermedad de Croker y Hartzell, Granuloma séptico, Granuloma hemangiomatoso, Hemangioma lobular capilar, Hemangioma capilar eruptivo, etc. Hartzell acuño el término de "Granuloma piógeno" o "Granuloma pyogenicum" en 1904; sin embargo, este nombre es considerado equivocado, debido a que el GP no produce pus, y no representa un verdadero granuloma (1).

La etiología del GP aún no está muy clara, se considera que es una lesión reactiva a varios estímulos de bajo grado, entre los cuales podemos contar traumas repetidos (2), agresiones, factores hormonales (3) y ciertos fármacos (4). Alrededor del $2 \%$ de las mujeres embarazadas desarrollan una GP intraoral en los primeros cinco meses de embarazo (1); la alta incidencia de esta lesión durante el embarazo se asocia con altos niveles de estrógeno y progesterona $(3,5)$. Además, se considera el tipo más común de hiperplasia en la boca (37\% de todas las lesiones reactivas), con una mayor prevalencia en mujeres (1.5:1) y presencia de factores etiológicos locales en $16 \%$ de todos los casos (6).

Clínicamente el GP oral aparece como una lesión exofitica, habitualmente pediculada, de superficie lobulada y tonalidad rojiza. Puede estar ulcerado y muestra una gran propensión al sangrado. $\mathrm{Su}$ ubicación principal son las encías: $75 \%$ de todos los casos (6). Puede aparecer con menos frecuencia en los labios (7), la lengua $(8,9)$, reborde alveolar (1), paladar (10), o estar asociado a otras patologías (11). Generalmente no produce resorción ósea, ni dentaria, aunque se ha reportado resorción radicular externa, asociado a GP (12).

El GP múltiple, es un fenómeno documentado, pero no común, ocurre después de una reacción de hipersensibilidad asociado al uso de drogas, como la carbamacepina, ciclosporina $(8,11)$, fenitoina, nifedipino (11), uso de retinoides, quemaduras, embarazo, presencia de anticuerpos antifosfolipidos, o enfermedad hepática (13). Además, se relacionan con agentes antirretrovirales, inhibidores epidérmicos, anticonceptivos, rituximab, levotiroxina, etc. (14).

Tradicionalmente, el tratamiento de elección para este tipo de lesiones es la exeresis quirúrgica con curetaje subperióstico, para prevenir la recurrencia deben eliminarse los factores irritantes potenciales (sarro, restauraciones rebosantes, etc.). Si a pesar de estas medidas ocurre la recurrencia se optará por tratamientos alternativos o complementarios.

El presente caso clínico tiene como objetivo reportar la presencia de GP múltiple y gigante en una paciente hipotiroidea en tratamiento con levotiroxina, y plantear la posibilidad de que este medicamento haya actuado como agente etiológico, en vista que no existen reportes sobre GP múltiple en cavidad oral asociados al uso de levotiroxina. 


\section{Reporte de caso}

Paciente de sexo femenino, ama de casa, de 51 años, con antecedentes de Psoriasis eritrodermica descamativa generalizada desde el nacimiento, en tratamiento tópico con retinoides, corticoides, y antihistamínicos; hipotiroidismo, en tratamiento con levotiroxina sódica $(0.05 \mathrm{mg} /$ día $)$ hace 2 años; RAM a múltiples medicamentos: quinolonas (ciprofloxacino, levofloxacino), penicilina, cefalexina (keflex), cloranfenicol, aminoglucosidos (amikacina, gentamicina), pseudoefedrina, AINES, lidocaína; ICC diastólica ventricular controlada; ITU a repetición controlada; hipoacusia neurosensorial.

Fue referida a la unidad de Cirugía Bucomaxilofacial por presentar aumento de volumen en reborde alveolar de ambos maxilares que limitaban su función masticatoria; tiempo de enfermedad aproximadamente 18 meses, inicio insidioso, curso progresivo.

Al examen clínico extraoral se observó placas costrosas descamativas en piel de todas las regiones faciales. Al examen intraoral, apertura bucal conservada, higiene oral deficiente, movilidad dentaria generalizada, encías edematosas, congestivas, en el primer cuadrante se evidenció lesión tumoral lobulada, de forma regular, bordes definidos, ulcerada, base sésil, color violáceo, de aproximadamente 2.5 x $2.5 \mathrm{~cm}$, asociada a pieza 1,5 , la cual presentaba movilidad grado II-III; en el tercer cuadrante, edentulo, se evidenció lesión tumoral lobulada, de forma regular, bordes definidos, ulcerada, base sésil, color violáceo, de aproximadamente $1.5 \times 1 \mathrm{~cm}$; y en el cuarto cuadrante lesión tumoral lobulada, de forma regular, bordes definidos, ulcerada, base sésil, color violáceo, de aproximadamente $1 \times 0.6 \mathrm{~cm}$, asociada a piezas dentarias 4,5 y 4,8 , las cuales presentaban movilidad dentaria grado II-III (Figura 1,2,3).

En la radiografía panorámica se observó pérdida ósea generalizada debida a enfermedad periodontal, sin áreas de erosión a nivel de las lesiones, leve radiopacidad correspondiente a las masas tumorales en el primer, tercer y cuarto cuadrantes (Figura 4).

Granuloma piógeno, granuloma periférico de células gigantes, y fibroma irritativo múltiple fueron considerados en el diagnostico presuntivo, no se incluyeron neoplasias malignas por las características clínico radiográficas de la lesión, y el tiempo de enfermedad.

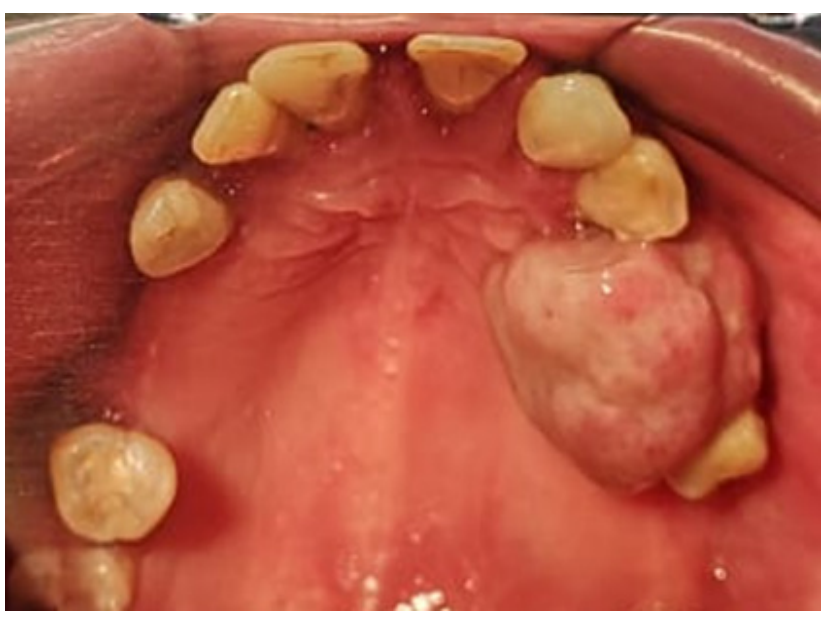

Figura 1. Granuloma Piógeno múltiple, en cuadrantes I, III, y IV

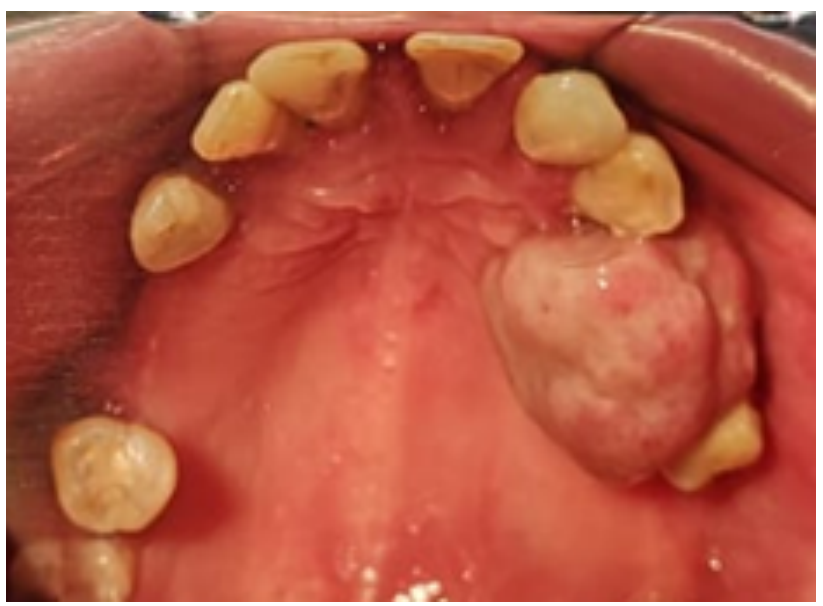

Figura 2. Detalle de Granuloma Piógeno en I cuadrante

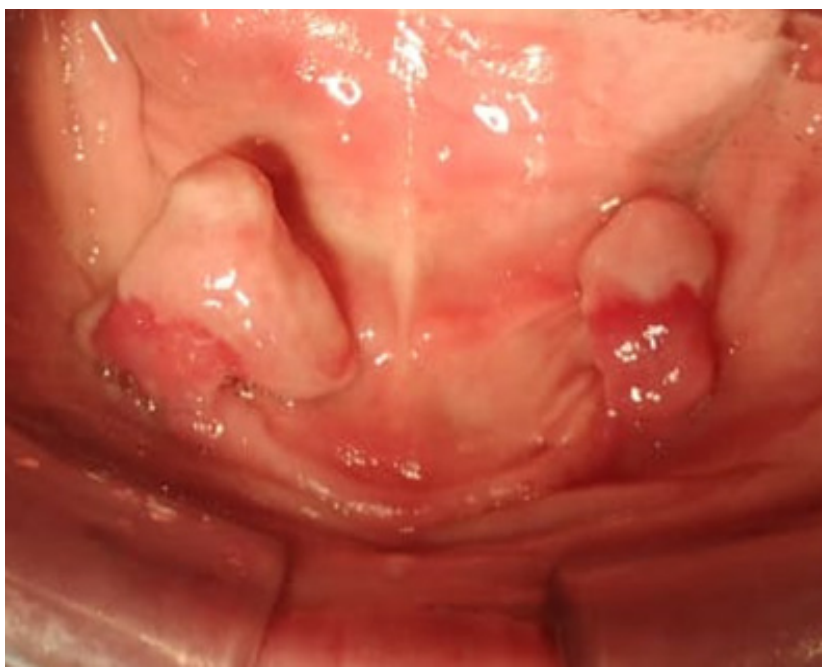

Figura 3. Detalle de Granuloma Piogeno en III y IV cuadrantes 


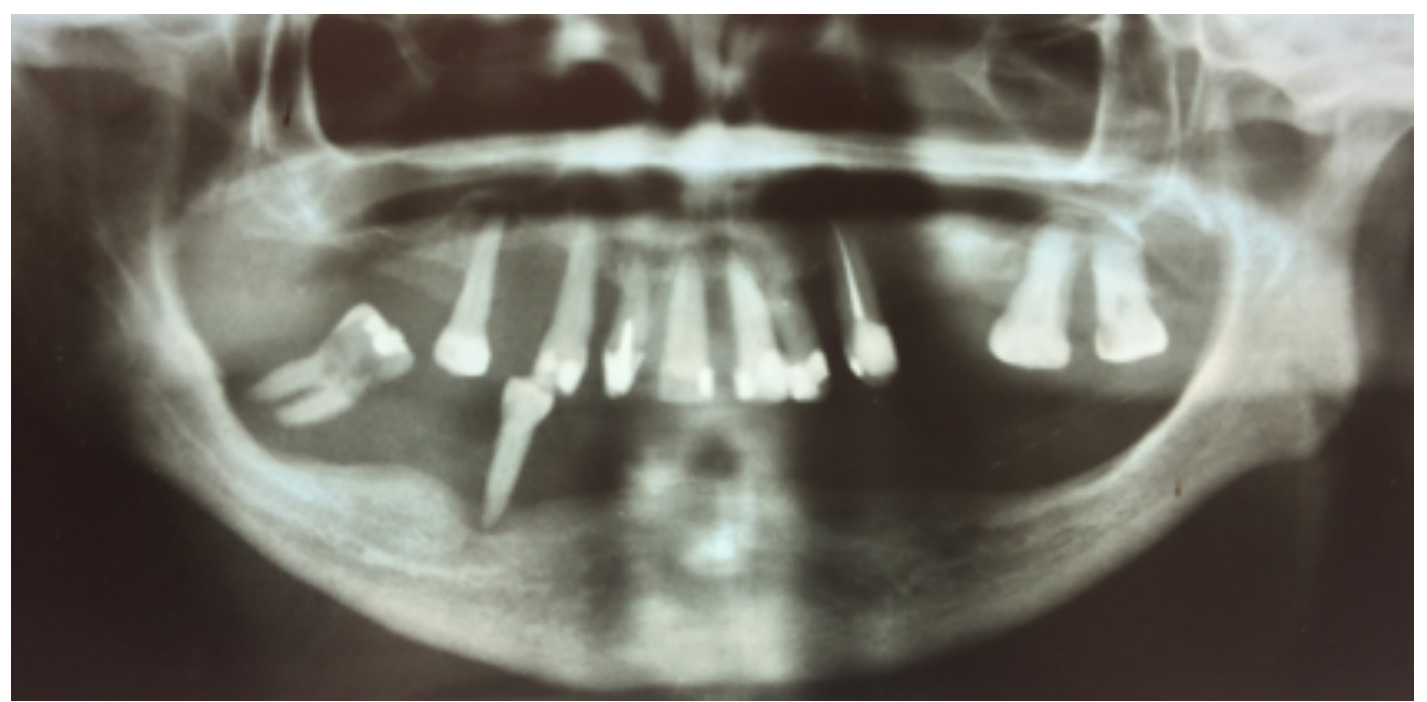

Figura 4. Ortopantografia preoperatoria

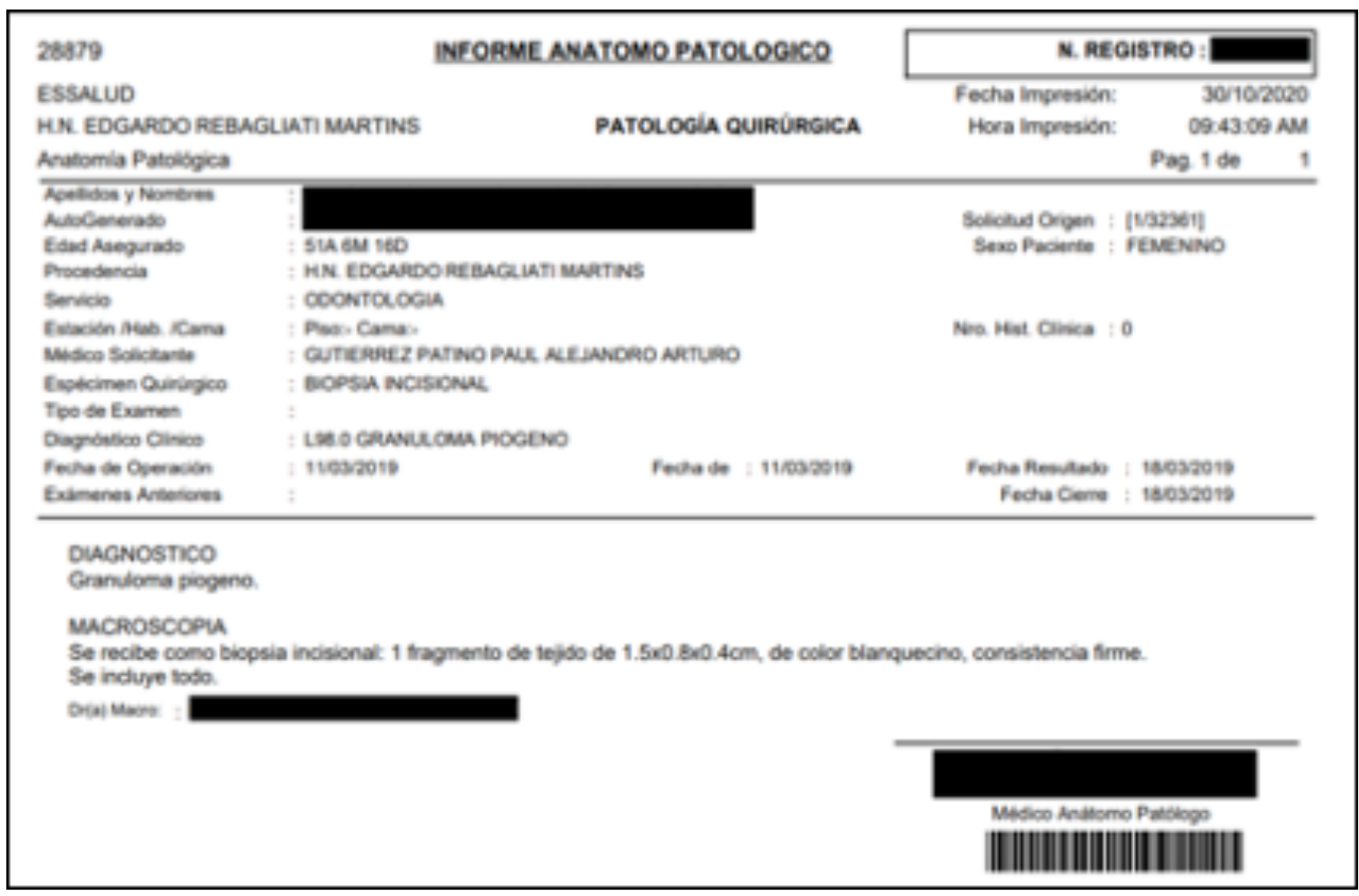

Figura 5. Informe Anatomopatologico

Se planificó el tratamiento en dos fases, bajo anestesia local, y previa interconsulta a los servicios de alergia para prueba de provocación a mepivacaina al 3\% sin epinefrina (informó que paciente tolera anestésico) y endocrinología, que confirmó el control adecuado de su hipotiroidismo.
En la primera fase se realizó la exodoncia de las piezas 4,5 y 4,7, exeresis de las lesiones en el tercer y cuarto cuadrantes y curetaje subperiostico. En la segunda fase se realizó la exodoncia de la pieza 1,5, exeresis de la lesión del primer cuadrante y curetaje subperiostico, además se planteó a la paciente la 


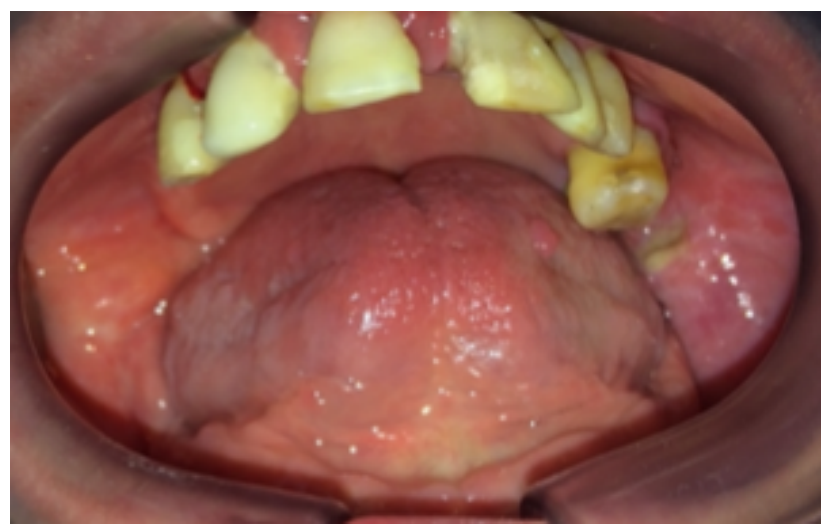

Figura 6. Control a los tres meses

exodoncia de las piezas restantes, por presentar enfermedad periodontal (irritante potencial), pero la paciente se negó, asumiendo la posibilidad de recurrencia de la lesión. Las lesiones se enviaron a anatomía patológica, confirmándose el diagnóstico definitivo de GP (figura 5).

La paciente se encuentra en el tercer mes post operatorio, presenta ausencia de sintomatología, y no hay evidencia de signos de recurrencia (Figura 6).

\section{DISCUSIÓN}

Originalmente, se creía que los GP eran infecciones botriomicóticas, que se transmitían de caballo a hombre. Posteriormente, se propuso que estas lesiones son causadas por algunas bacterias piógenas como estreptococos y estafilococos. Sin embargo, no hay evidencia de ningún organismo infeccioso aislado de las lesiones que confirme la relación improbable con alguna infección y, por lo tanto, el nombre es un nombre inapropiado $(8,15)$. Se cree que la infección bacteriana no específica es una afectación secundaria en lugar de ser la principal etiología de esta lesión (16).

Shamim etal., en un análisis de 244 casos de lesiones gingivales, descubrió que las lesiones no neoplásicas representaban el $75.5 \%$ de los casos, siendo el GP oral la lesión más frecuente, representando el 52.71\% de los casos (12). Estas lesiones se limitan a la encía y rara vez involucran el hueso alveolar (15). Aunque a veces, puede estar asociada con la erosión ósea de la corteza labial $(1,17)$, especialmente los GP de larga data.
El curso natural de esta lesión se puede clasificar en tres fases distintas, a saber, fase celular: se parecen al color normal de la mucosa; fase capilar / fase vascular: son rojizas a púrpura debido al aumento de la vascularización; y fase involutiva: masa de color rosado a blanquecino (16). En el caso presentado, se trataba de lesiones exofiticas, color violáceo, según la clasificación se encontrarían en la segunda fase.

El GP se presenta en cavidad oral, pero también se muestra en nasofaringe (18), el intestino delgado, colon, recto, cicatrices de quemaduras (19), además se ha reportado en lechos ungueales de dedos de manos y pie (14).

De los tres GP reportados, el del maxilar superior medía más de $2.5 \mathrm{~cm}$, la literatura considera a estos GP como "gigantes" $(9,15)$; y se sugirió que algunos casos están relacionados con pacientes sistémicamente comprometidos, aunque se encontraron publicaciones de GP gigantes en pacientes sanos, incluido gestantes (19).

La imagen microscópica del GP muestra un tejido de granulación exuberante que está cubierto por un epitelio atrófico/hiperplásico que puede ulcerarse a veces y revela exudados fibrinosos, también se observa la presencia de infiltración celular inflamatoria mixta (16). En base a esto, esta lesión se puede clasificar en dos grupos: cuando se encuentra que los vasos capilares están organizados en los lóbulos granulomatosos rodeados por una delgada banda de colágeno, la formación se denomina "hemangioma capilar lobular" (LCH), mientras que cuando las formaciones vasculares se entrelazan en el tejido sin orden aparente, se denomina "hemangioma capilar no lobular" (no LCH) $(6,14)$.

El aumento de los niveles de estrógenos y progesterona durante el embarazo puede tener dos efectos en la patogénesis de GP, las hormonas no solo mejoran la expresión de factores angiogenicos activados por monocitos y macrófagos en el tejido inflamado, también disminuye apoptosis de macrófagos de células de granuloma para extender el efecto angiogenico (3).

Entre los antecedentes sistémicos que refiere la paciente, nos llama la atención la Psoriasis eritrodermica descamativa generalizada desde el nacimiento, en tratamiento tópico con retinoides; $\mathrm{y}$ 
el Hipotiroidismo, en tratamiento con levotiroxina sódica $(0.05 \mathrm{mg} /$ día). Se ha reportado el GP cutáneo en relación al tratamiento con retinoides para la Psoriasis $(13,20)$, se considera que los retinoides disminuyen las uniones entre los queratinocitos; también tienen propiedades angiogénicas e inhiben las colagenasas y las gelatinasas (14); sin embargo, la paciente no manifestó la presencia de este tipo de lesiones en piel. No obstante, entre las características clínicas de la Psoriasis se describe la lengua geográfica (70\%), asociada en $75 \%$ de los casos a lengua fisurada (21); lesiones que no mostraba la paciente.

Las drogas son una causa bien conocida de desarrollo de GP, están relacionados en su mayoría con el uso de retinoides, agentes antirretrovirales, inhibidores del receptor del factor de crecimiento epidérmico (EGFR), y drogas antitumorales (14), en la tabla 1 se presenta el mecanismo de acción de una serie de drogas que inducen a la formación de GP. En la revisión de la literatura, solo fue reportado un caso de GP cutáneo (subungueal) múltiple asociado al uso de Levotiroxina (14), aunque fue de localización extraoral, las características clínicas e histopatológicas son similares.

El GP puede estar asociada con la presencia de trastornos metabólicos y tiroideos $(11,22)$, especialmente las que se presentan en localizaciones inusuales (9). Algunos factores como el óxido nítrico sintasa, el factor de crecimiento endotelial vascular, el factor de crecimiento de fibroblastos y el factor de crecimiento del tejido conectivo, son conocidos por estar envueltos en la angiogénesis y en el crecimiento rápido del granuloma piógeno (23).

Las hormonas tiroideas, particularmente T4, demostraron ser los mediadores de la angiogénesis y proliferación tumoral. A este respecto, la levotiroxina usada por la paciente desde hace más de dos años, podría ser un posible factor etiológico en los GP a través de efectos proangiogénicos y proliferativos. Sin embargo, el mecanismo patógeno determinado sigue siendo desconocido (15). Recientemente, se ha descubierto que varias proteínas son determinantes importantes en la morfogénesis vascular, entre ellas Tie-2, angiopoyetina-1 (Ang-1), angiopoyetina-2 (Ang-2), efrina-B2 y Eph-B4, las cuales muestran sus funciones en la angiogénesis y el remodelado de vasos sanguíneos en el estadio embrionario $(9,24)$. Estos cinco polipéptidos, aumentados en el GP, desempeñan un papel importante en la desregulación del balance angiogénico y antiangiogénico, lo que propicia el desarrollo de una considerable neovascularización en esta lesión (9).

Tabla 1. Mecanismo de acción de algunas drogas que inducen Granulomas Piógenos

\begin{tabular}{ll}
\hline \multicolumn{1}{c}{ Droga } & $\begin{array}{l}\text { Momologías entre las secuencias de aminoácidos de la proteína 1 de unión al ácido retinoico y } \\
\text { el sitio catalizador del VIH-1. Inhibición de las proteasas endógenas }\end{array}$ \\
\hline Indinavir & Proangiogénico \\
5-fluorouracilo & $\begin{array}{l}\text { liberación de factores angiogénicos estimulados por el proceso inflamatorio y deterioro de las } \\
\text { funciones hepáticas }\end{array}$ \\
Carbamacepina & Efecto secundario vascular \\
Rituximab & $\begin{array}{l}\text { Disminuye la unión entre los queratinocitos y también tienen propiedades angiogénicas e } \\
\text { inhiben las colagenasas y gelatinasas }\end{array}$ \\
Retinoides & $\begin{array}{l}\text { Efecto parecido al de los retinoides debido a las homologías entre los receptores retinoides y } \\
\text { sitio catalítico de la proteasa del VIH-1 }\end{array}$ \\
Drogas Antivirales & Efecto secundario vascular \\
Imatinib & Sobre expresión de MAP quinasa \\
Vemurafenib & Expresión de queratinocitos de la familia del EGF y EGFR, aumento de la liberación de \\
Terapia anti TNF- $\alpha$ & VEG
\end{tabular}

(Modificado de Kemal et. al. 2015) 
Los análogos de la hormona tiroidea son proangiogénicos (incluida T4), la actividad proangiogénica de estos análogos parece estar en un receptor de la superficie celular para la hormona, la integrina $\alpha v \beta 3$ (la T4 actúa sobre la integrina plaquetaria $\alpha v \beta 3$ ), que conduce a la transducción de la señal hormonal por MAPK (ERK 1/2), en consecuencia, se produce la transcripción del gen del factor de crecimiento de fibroblastos básico (bFGF). El gen del factor de crecimiento endotelial vascular (VEGF) también puede transcribirse en respuesta a la unión de la hormona tiroidea por el receptor de la integrina. Finalmente, se ha demostrado que la acumulación de Ang-2, pero no de Ang-1, se produce en células endoteliales estimuladas por análogos de la hormona tiroidea a través del receptor de la superficie celular (25). Se cree que Ang-1 funciona mediante la unión y fosforilación de su receptor tirosina quinasa Tie-2, y estaría involucrado en una etapa posterior de la angiogénesis, así como en la estabilización de los vasos sanguíneos existentes al modular las interacciones entre las células endoteliales y las células de soporte circundantes. Ang-2 también se une a Tie-2 pero no lo fosforila y se cree que actúa como antagonista de Ang-1 (26).

La familia de transducción de señales y activador de la transcripción (STAT), como STAT1 $\alpha$ y STAT3, también son fosforiladas en serina por ERK 1/2 activada por hormona tiroidea. Las proteínas STAT están implicadas en la bioquímica del factor de crecimiento vascular, y así mejorar la angiogénesis. Es decir, un efecto de la hormona iniciado en la superficie celular y que no implica necesariamente el acceso de la hormona al interior de la célula, culmina en la expresión génica relevante para la neovascularización (25). Este mecanismo explicaría el papel de la levotiroxina (T4 sintética) en la proliferación de GP múltiples, en ambos maxilares. En informes anteriores sobre GP inducidos por fármacos, se demostró que el inicio de GP fue menos de 16 meses después del uso del presunto fármaco incitador (14), en el caso presentado la paciente tomaba levotiroxina seis meses antes que se manifiesten las lesiones.

En vista de sus características clínicas, el diagnóstico diferencial de GP incluye granuloma periférico de células gigantes, fibroma osificante periférico, metástasis de tumores malignos, hemangioma, hiperplasia inflamatoria, sarcoma de
Kaposi, angiosarcoma y linfoma no Hodgkin (4). Además, TUSGE (granuloma traumático ulcerativo con eosinofilia estromal) (9). El diagnóstico definitivo depende del resultado anatomopatologico.

Los GP pueden tratarse mediante cirugía, crioterapia, cauterización $(6,14)$. Otras formas de tratamiento incluyen láser Nd-YAG (granate de itrio y aluminio dopado con impurezas de neodimio), láser de colorante pulsado con lámpara de flash, inyección intralesional de etanol o corticosteroides y escleroterapia con tetradecil sulfato de sodio (STS) $(7,15)$.

La escisión incompleta, la imposibilidad de eliminar los factores etiológicos o el trauma repetido contribuyen a la recurrencia de estas lesiones. Se informa que la tasa de recurrencia es mayor para las lesiones extirpadas durante el embarazo, probablemente debido a la persistencia de las hormonas (1). Después de la escisión, la recurrencia ocurre en hasta el $16 \%$ de los casos. Debe enfatizarse que los casos gingivales muestran una tasa de recurrencia mucho más alta que las lesiones en otros sitios de la mucosa oral $(4,8)$.

Se concluye que, aunque el GP es generalmente una respuesta exagerada de los tejidos a irritantes locales, en el caso presentado a pesar que la paciente tenia mala higiene oral, este hecho no explica por si solo la aparición casi simultánea de estas lesiones en los tres cuadrantes de la cavidad oral, por lo tanto planteamos la interrogante acerca de si este medicamento ha sido el único agente etiológico del GP múltiple, o por lo menos, exacerbó la patología producida por los irritantes locales que esta mostraba; para dilucidar estas dudas se requieren mayores estudios; de comprobarse esta hipótesis, este el primer reporte de GP intraoral asociado a levotiroxina.

\section{Correspondencia}

Alejandro Arturo Gutiérrez Patiño-Paul Buenaventura Aguirre 293, Barranco, Lima, Perú. Teléfono: 51985790151

Correo electrónico: agutierrezpp3@hotmail.com

\section{REFERENCIAS BIBLIOGRAFICAS}

1. Mohapatra S, Singh K, Singh L, Kumar P. Oral pyogenic granuloma: A review. JODA. 2014;3(1):5-9. 
2. Patussi C, Sassi LM, Da Silva WP, Zavarez LB, Schussel JL. Oral Pyogenic Granuloma after Tongue Piercing Use: Case Report. Dentistry. 2014; 4: 229.

3. Yuan K, Wing L, Lin M. Pathogenetic roles of angiogenic factors in pyogenic granulomas in pregnancy are modulated by female sex hormones. J Periodontol. 2002;73(7):701-8.

4. Ghalayani P, Hajisadeghi S, Babadi F. Extragingival pyogenic granuloma associated with medication: Report of an unusual case. Dent Res J. 2014;11(3):4004.

5. Sharma S, Chandra S, Gupta S, Srivastava S. Heterogeneous conceptualization of etiopathogenesis: Oral pyogenic granuloma. Natl J Maxillofac Surg. 2019;10:3-7.

6. Gadea C, Cartagena A, Cáceres A. Oral pyogenic granuloma diagnosis and treatment: a series of cases. Rev Odont Mex. 2017;21(4):244-52.

7. Asha V, Dhanya M, Patil BA, Revanna G. An unusual presentation of pyogenic granuloma of the lower lip. Contemp Clin Dent 2014;5(4):524-6.

8. Peters S, Koslovsky D, Yoon A, Philipone E. Pyogenic Granuloma in the Tongue in a Five Year Old: A Case Report. J Clin Pediatr Dent. 2018;42(5):1-3.

9. González M, Piña C, Sánchez J. Granuloma piógeno de localización atípica: reporte de un caso. Rev. ADM. 2017;74(4):198-201.

10. Amirchaghmaghi M, Falaki F, Mohtasham N, Mozafari PM. Extragingival pyogenic granuloma: a case report. Cases J. 2008;1(1):371.

11. Ocampo K, Díaz L, Barrera J. Pyogenic granuloma associated with mandibular odontoma. J Oral Res 2014; 3(3): 168-72.

12. Suprith SS, Patil L, Thakur S. A rare case report of pyogenic granuloma associated with external root resorption. J Interdiscip Dentistry. 2019;9:39-43.

13. Palmero M, Pope E. Eruptive pyogenic granulomas developing after drug hypersensitivity reaction. J Am Acad Dermatol. 2009;60(5):855-7.

14. Kemal M, Yosma E, Oguzhan I, Simsek T, Hwan T. Multiple Subungual Pyogenic Granulomas Following Levothyroxine Treatment. J Craniofac Surg. 2015; 26(6):476-7.

15. Mubeen K, Vijayalakshmi K. Abhishek R. Oral pyogenic granuloma with mandible involvement: An unusual presentation. J. Dent. Oral Hyg. 2011;3(1):69.
16. Marla V, Shrestha A, Goel K, Shrestha S. The Histopathological Spectrum of Pyogenic Granuloma: A Case Series. Case Rep Dent. 2016;1:1-6.

17. Mohanty G, Mohanty R, Satpathy A. Simultaneous occurrence of pyogenic granuloma at multiple sites associated with bone loss: Report of a rare case. J Indian Soc Periodontol. 2018;22(2):174-177.

18. Assiri K, Al-Ahmari M. A rare case report of pyogenic granuloma of nasopharynx. Int J Otorhinolaryngol Head Neck Surg. 2019;5(4):1091-94.

19. Limmonthol S, Sayungkul C, Klanrit P. Oral pyogenic granuloma presenting as an atypically large soft tissue mass: A case report. J Oral Maxillofac Surg Med Pathol. 2014;26:258-261.

20. Liu J, Zhou BR, Yi F, Wu HJ, Zhang JA, Luo D. Pyogenic granuloma in a patient with psoriasis successfully treated by 5-aminolevulinic acid photodynamic therapy: A case report. Exp Ther Med. 2016;11(1):345-7.

21. Picciani B, Santos V, Teixeira-Souza T, et al. Investigation of the clinical features of geographic tongue: unveiling its relationship with oral psoriasis. Int J Dermatol. 2017;56(4):421-7.

22. Anitua E, Piñas L. Pyogenic granuloma in relation to dental implants: Clinical and histopathological findings. J Clin Exp Dent. 2015;7(4):447-50.

23. Cordero K, Pardo N, Arellano A. Granuloma piógeno de presentación inusual: Reporte de caso. Odont. Vital. 2016; 25:35-42.

24. Yuan K, Jin Y, Lin M. Expression of Tie-2, angiopoietin-1, angiopoietin-2, ephrinB2 and EphB4 in pyogenic granuloma of human gingiva implicates their roles in inflammatory angiogénesis. J Periodont Res. 2000;35:165-71.

25. Davis P, Davis F, Mousa S. Thyroid Hormone-Induced Angiogenesis. Curr Cardiol Rev. 2009; 5:12-16.

26. Mitchell J, Parangi S. Angiogenesis in Benign and Malignant Thyroid Disease. Thyroid. 2005; 15(6):494510 .

Recibido : 15-04-2020

Aceptado : 15-11-2020 